\title{
Waldenström's macroglobulinaemia secreting a paraprotein with lupus anticoagulant activity: Possible association with gastrointestinal tract disease and malabsorption
}

\author{
R C Tait, P K Oogarah, J B Houghton, S E Farrand, M R Haeney
}

Department of R C Tait

J B Houghton Pathology

P K Oogarah

Department of

Immunology

S E Farrand

M R Haeney

Hope Hospital, Eccles Old Road, Salford M6 8HD

Correspondence to: R C Tait

Accepted for publication 26 January 1993 Haematology

Department of

\begin{abstract}
A 51 year old man with Waldenström's macroglobulinaemia presented with a malabsorptive syndrome related to extensive small bowel lymphangiectasia caused by immunoglobulin accumulation. The patient's plasma had strong lupus anticoagulant activity and the IgM $\lambda$ paraprotein displayed specificity for the negatively charged phospholipids phosphatidyl serine and phosphatidyl inisitol, as well as the neutral phosphatidic acid. Despite treatment for the macroglobulinaemia the patient died and at necropsy was found to have myocardial ischaemia and segmental infarcts in the spleen and kidney. The coexistence of these relatively rare findings suggests a possible association between Waldenström's macroglobulinaemia with gastrointestinal manifestations and paraprotein specificity for phospholipid.
\end{abstract}

(F Clin Pathol 1993;46:678-680)

Lupus anticoagulants, although first described in systemic lupus erythematosus, ${ }^{1}$ have been identified in a variety of unrelated conditions. ${ }^{2}$ The antibodies responsible for the lupus anticoagulant effect are usually polyclonal, but a few instances of lupus anticoagulant associated with monoclonal gam-

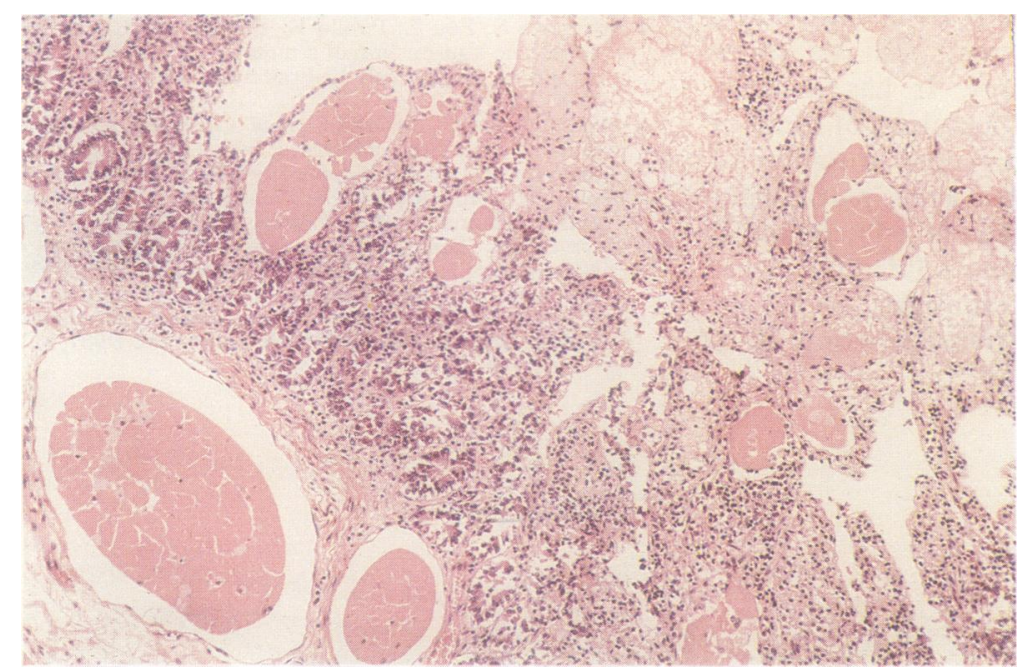

Figure 1 Mucosal folds of the duodenum showing dilated mucosal and submucosal lymphatics containing homogeneous eosinophilic coagulum. mopathy have been reported. ${ }^{23}$ In these cases, however, the clinical sequelae related to the lupus anticoagulant are often minimal. We report a case of Waldenström's macroglobulinaemia (WM) in which the IgM paraprotein had phospholipid specificity and in which necropsy showed infarcts in several organs. The patient also had unusual gastrointestinal manifestations of the disease.

\section{Case report}

A 51 year old man presented with an eight month history of malaise, weight loss, and worsening diarrhoea which was predominantly nocturnal. He was a lifelong smoker, was receiving no medication, and denied any haemostatic problems or symptoms of ischaemic heart disease. His spleen was enlarged (18 cm in axial length), but the only demonstrable lymphadenopathy, detected by ultrasound scan, was in the porta hepatis and para aortic area. The haemoglobin concentration was $109 \mathrm{~g} / \mathrm{l}$, platelet count $197 \times 10^{9} / \mathrm{l}$, and the white cell count $37 \cdot 2 \times 10^{\%} / 1$ with $89 \%$ lymphocytes and lymphoplasmacytoid cells. Serum IgM was increased at $60 \mathrm{~g} / 1$ and qualitative analysis showed the presence of an $\operatorname{IgM} \lambda$ paraprotein in the serum and free $\lambda$ light chains in the urine. Renal and liver function tests were normal. Faecal fat was increased at $40.5 \mathrm{mmol} /$ day (normal $0-17$ mmol/day). At gastroscopy the stomach was normal but the duodenal mucosa had an abnormal feathery appearance with scattered white deposits. Biopsy specimens from these areas showed focal clubbing of villi due to lymphangiectasia with dilated lymphatics containing homogeneous eosinophilic coagulum (fig 1). Groups of foamy histiocytes were present in the lamina propria but there was no significant lymphoid infiltrate. Bone marrow was hypercellular due to a diffuse, predominantly small cell, lymphoid infiltrate with only occasional plasma cells. Mast cells were not prominent.

Prothrombin time (32 seconds, control 14 seconds) and partial thromboplastin time (110 seconds; control 40 seconds) were abnormal but thrombin clotting time and fibrinogen were normal. In vitro mixing tests and also parenteral administration of vitamin $\mathrm{K}$ resulted in partial correction of the prothrombin time to 20 seconds but had no effect on the apparent intrinsic defect. Individual assays of factors II, VIII, IX and X 
all gave abnormally low results and both kaolin clotting time (ratio 1.66) and the Dilute Russell's Viper Venom Test (ratio 2.4; shortening to 1.4 with a platelet correction procedure) were highly suggestive of a lupus anticoagulant. The $\operatorname{IgM}$ anticardiolipin titre was 46000 units $/ \mathrm{ml}$ (normal $<5 \mathrm{U} / \mathrm{ml}$ ).

Treatment with prednisolone, $(60 \mathrm{mg} /$ day) and weekly cyclophosphamide $(500 \mathrm{mg})$ resulted in a modest reduction in IgM titre but no improvement in symptoms and lupus anticoagulant activity remained strongly positive until his sudden death six weeks later. Necropsy showed ischaemic heart disease, an enlarged spleen $(760 \mathrm{~g})$, and segmental infarcts in spleen and kidney. The small bowel mucosa had a "velvety" white appear-

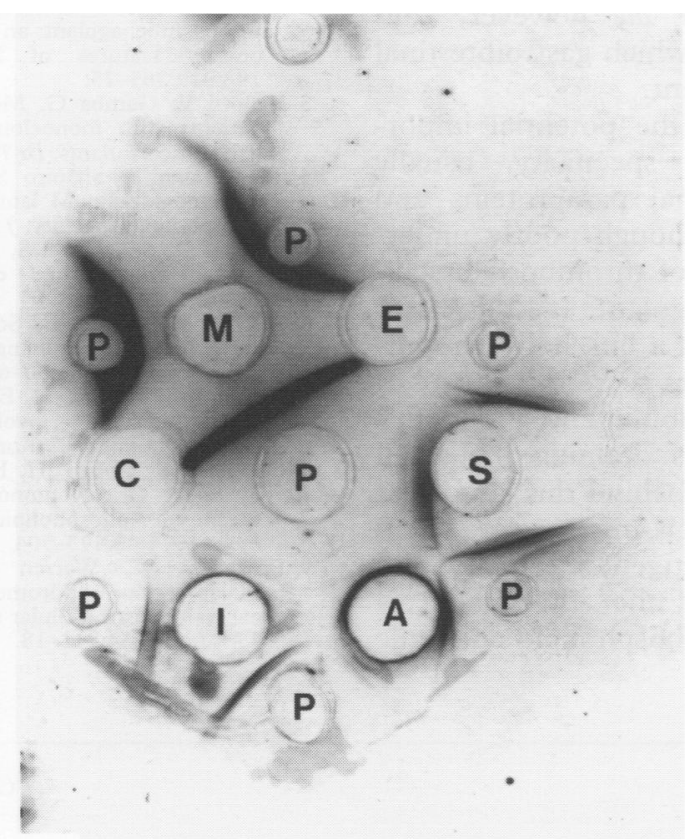

(A)
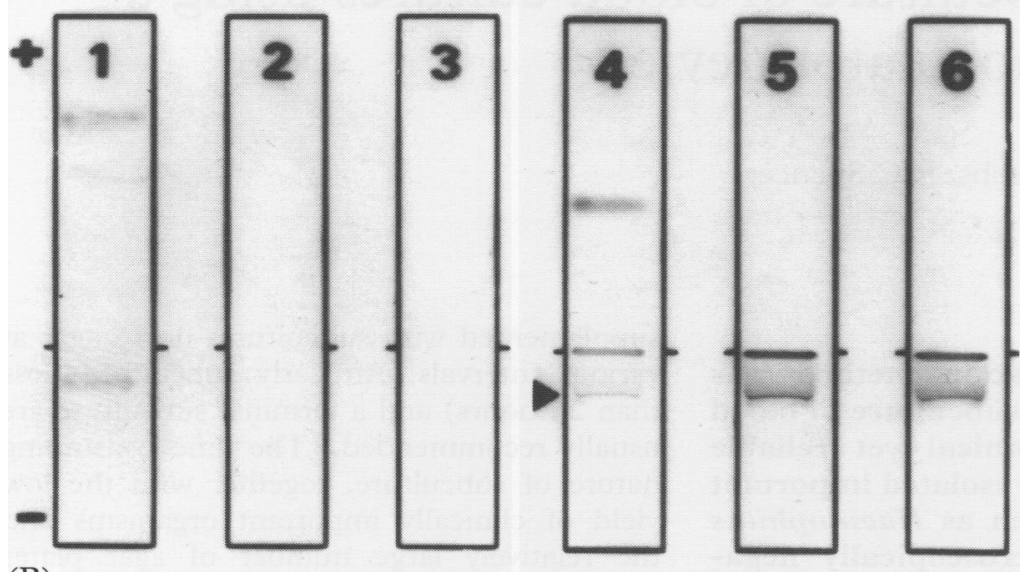

(B)

Figure 2 The Ouchterlony plate $(A)$ shows dark precipitin lines between wells containing patient's serum $(P)$ and wells containing anti-IgM (M), and also between patient's serum and wells containing sonicates of phosphatidyl serine (S), phosphatidyl inisitol (I), and phosphatidic acid (A), but not phosphatidyl choline (C) or phosphatidyl ethanolamine (E).

Electrophoretic strips (B) Nos 1-3 are from a control patient with Waldenström's macroglobulinaemia and a similar titre of monoclonal IgM as the patient's serum which is run in strips Nos 4-6. Strips 1 and 4 are fixed for protein and therefore show an anodally migrating albumin band and a slow cathodally migrating paraprotein band (arrowed). The other strips are overlayed with phospholipid sonicates (phosphatidyl serine-Nos 2 and 5; phosphatidyl inisitol Nos 3 and 6) for 15 minutes before washing and then staining for protein. The only remaining protein being that which has been immunofixed by the phospholipid. ance and histological results were similar to the antemortem duodenal biopsy specimen appearances. The mesentery contained white streaky patches (lymphatics) and nodules (lymph nodes) engorged with amorphous, eosinophilic extracellular material, presumably immunoglobulin. Many regional draining lymph nodes showed an extensive loss of normal architecture with accumulation of similar extracellular material and little remaining lymphoid material. The eosinophilic extracellular material was periodic acid Schiff positive, diastase resistant, and did not stain for amyloid but showed an admixture of phospholipid on histochemistry (bromination-acetone-Sudan black). A few lymph nodes showed a diffuse lymphoplasmacytoid infiltrate displaying methyl green pyronin positivity. Similar cells were present in the spleen, bone marrow, and portal tracts of the liver.

\section{IMMUNODIFFUSION AND PRECIPITATION} STUDIES

In an attempt to show that the IgM paraprotein had phospholipid specificity an Ouchterlony immunoprecipitation plate was set up with wells containing dilutions of the patient's serum and other wells containing sonicates of specific phospholipids prepared according to the methods of Thiagarajan et $a l .{ }^{4}$ Precipitin lines were obtained between the serum and the two negatively charged phospholipids, phosphatidyl serine and phosphatidyl inisitol, and also the neutral phosphatidic acid, but not phosphatidyl choline or phosphatidyl ethanolamine (fig 2A). Furthermore, absorption of the patient's serum by anti- $\lambda$ abrogated all precipitation reactions. Overlaying electrophoretic strips from the patient's serum with the same phospholipid preparations as above showed that both phosphatidyl serine and phosphatidyl inisitol were immunofixed at the exact point of migration of this patient's paraprotein but that no immunofixation occurred with the IgM paraprotein from a different patient with Waldenström's macroglobulinaemia (fig 2B).

\section{Discussion}

Disease of the small intestine in Waldenström's macroglobulinaemia is rare, but a few cases, manifest by malabsorption and diarrhoea, have been described. ${ }^{5-7}$ Indeed, the case reported here has almost identical clinical, pathological, and histological features, particularly the striking "velvety" mucosa and lymphatic dilatation with amorphous eosinophilic material. Of lesser rarity is the finding of a monoclonal paraprotein in some individuals with a lupus anticoagulant. ${ }^{2-4}$ In only a few cases, however, has the paraprotein been shown to display phospholipid specificity. ${ }^{348}$ Despite the well recognised association between lupus anticoagulant and thrombosis, and in contrast to the case reported here, few of these patients previously described seem to have had thrombotic complications. 
It is interesting to note that one of the early reports of small intestinal disease in Waldenström's macroglobulinaemia ${ }^{5}$ describes a patient who also had abnormal coagulation tests, probably false positive syphilis serology, and who sustained a cerebral artery thrombosis one year after diagnosis of Waldenström's macroglobulinaemia. Two other reports of intestinal Waldenström's macroglobulinaemia also noted abnormal coagulation and false positive syphilis serology, ${ }^{59}$ and in the second of these phospholipid was found in association with the extracellular immunoglobulin in the small intestinal lymphatics. Unfortunately, most reports of lymphoma associated paraproteins with lupus anticoagulant activity or phospholipid specificity do not document extent or localisation of disease. Cooper et al, however, ${ }^{8}$ did describe such a case in which gastrointestinal symptoms were prominent.

This case highlights the potential importance of the antigen specificity, usually unknown, of monoclonal paraproteins and also the ever present, though poorly understood, paradoxical risk of thrombosis associated with lupus anticoagulant. It leads us to suggest the possibility of a link between lymphoproliferative disorders secreting phospholipid specific immunoglobulin and a tendency to affect the lymphatics draining the small bowel. The mechanism behind this is unclear but we speculate that this might relate to an association between antigen specificity and recirculation patterns of the abnormal lymphoid clone. The high phospholipid antigenic load to which gut associated lymphoid tissue is exposed would increase the chances of a lymphoproliferative disorder arising here and having some degree of phospholipid specificity. Alternatively, lymphomas with phospholipid specificity arising elsewhere may localise in the lymphatic draining system of the small bowel due to a high content of the antigen in these areas.

We thank Dr KJ Stevenson at the UK Reference Laboratory for Anticoagulant Reagents and Control, Withington Hospital, Manchester, for the kind donation and preparation of the various phospholipid preparations.
ons

1 Conley CL, Hartmann RC. A haemorrhagic disorder caused by circulating anticoagulant in patients with disseminated lupus erythematosus. F Clin Invest 1952, 31:621-2.

2 Gastineau DA, Kazmier FJ, Nichols WL, Bowie EJW. Lupus anticoagulant: an analysis of the clinical and laboratory features of 219 cases. $\mathrm{Am} \mathcal{F}$ Hematol 1985;19:265-75.

3 Bellotti V, Gamba G, Merlini G, et al. Study of three patients with monoclonal gammopathies and 'lupuspatients with monoclonal gammopathies and 'lupu
like' anticoagulants. Br ₹ Haematol 1989;73:221-7.

4 Thiagarajan P, Shapiro SS, De-Marco L. Monoclonal immunoglobulin $M$ lambda coagulation inhibitor with phospholipid specifity. $\mathcal{f}$ Clin Invest 1980;66:397-405.

5 Cabrera A, de la Pava S, Pickren JW. Intestinal localisation of Waldenström's disease. Arch Intern Med 1964; 114:399-407.

6 Harris M, Burton IE, Scarffe JH. Macroglobulinaemia and intestinal lymphangiectasia: a rare association. $\mathscr{f}$ Clin Pathol 1983;36:30-6.

7 Bedine MS, Yardley JH, Elliott HL, Banwell JG, Hendrix TR. Intestinal involvement in Waldenström's macroglobulinemia. Gastroenterology 1973;65:308-15.

8 Cooper MR, Cohen JH, Huntley CC, Waite BM, Spees L, Spurr CL. A monoclonal IgM with antibodylike specifity for phospholipids in a patient with lymphoma. specifity for phospholipids

9 Pruzanski W, Warren RE, Goldie JH, Katz A Malabsorption syndrome with infiltration of the intestinal wall by extracellular monoclonal macroglobulin. $\mathrm{Am}$ f Med 1973;54:811-18.
Department of Bacteriology, Royal Hallamshire Hospital, Glossop Road, Sheffield S10 2JF

KJ Thickett

D J W Dabbs

R C Spencer

Accepted for publication

20 January 1993

\begin{abstract}
A multipoint inoculation method was used for the terminal subculture of blood cultures. This economical yet reliable technique successfully isolated important human pathogens such as Haemophilus influenzae from macroscopically negative blood cultures.
\end{abstract}

( Clin Pathol 1993;46:680-681)

Detection of bacteraemia is one of the most important functions of a clinical microbiology laboratory and accounts for a large part of laboratory workload. Traditional blood culture systems, involving enrichment broths and visual inspection of bottles, are usually supplemented with subcultures on to agar at various intervals. An early subculture (less than 24 hours) and a terminal subculture are usually recommended. ${ }^{1}$ The time consuming nature of subculture, together with the low yield of clinically important organisms and the relatively large number of agar plates required, has led some workers to conclude that terminal subculture of blood cultures may not be necessary. ${ }^{23}$ It has been shown, however, that in many blood culture systems important human pathogens such as Haemophilus influenzae and Neisseria meningitidis produce no macroscopic signs of growth. ${ }^{4}$ In an effort to resolve these problems and to make the process of terminal subcultures more economical we used a multipoint inoculation method. 